\title{
Collaborative Mining in Multiple Social Networks Data for Criminal Group Discovery
}

\author{
Amin Milani Fard, Martin Ester \\ School of Computing Science, Simon Fraser University, BC, Canada V5A 1S6 \\ $\{$ milanifard, ester $\} @$ cs.sfu.ca
}

\begin{abstract}
The hidden knowledge in social networks data can be regarded as an important resource for criminal investigations which can help finding the structure and organization of a criminal network. However such network based analysis has not been studied in an applied way and remains mostly a manual process. To assist inspectors and intelligence agencies discover this knowledge, we defined a new problem and then proposed a framework for automated network data analysis and deduction approach from multiple social networks by converting to transaction dataset, applying association mining, and statistical methods. By applying a game theory concept in a multi-agent model, we try to design a policy for knowledge discovery and inference fusion. This approach enables police stations to build and deploy P2P applications through a unified medium for finding criminals relationship and identifying suspicious guys.
\end{abstract}

Keywords: Social Network Analysis, Criminal Network Discovery, Associacion Rule Mining, Multi-agent System

\section{INTRODUCTION}

Social network analysis is now gaining more attentions in knowledge discovery from databases (KDD) with its theoretical basis, methods, social network analysis software, and researchers. Generally the analysis of social networks involves graphs and complex networks modeling to study many complex human and natural phenomena [1], [2], [3], [4]. In such a graph structure relationships between network users, whether in a one-way communication channel such as email exchange, or a two-way such as telephone connection, is modeled by graph edges. On the other hand, event-based graphs offer a basis for obtaining the same benefits while dealing with dynamic data regarding time of happening relationship or an event.

A common problem in criminal investigation is finding groups of related people who might have cooperated in a crime. Gathering such knowledge about the structure of a criminal network is an important matter; however, criminal network analysis has not been studied well in an applied way and still regarded as a manual process. To provide police inspectors and intelligence agencies with useful knowledge about such connections, a P2P application through a unified medium for finding criminal's relationship can be deployed for identifying suspects.

\section{A. Contributions}

In this work we first define a new problem on event-based modeling of social networks information, and then propose an effective generic mining method in multiple social networks for group discovery. Our method for analyzing criminal groups varies in different ways from previous works such as mining from multiple sources, generic event modeling, and providing a ranked result fusion list. We also proposed a multi-agent method for knowledge extraction and inference combination.

The structure of the paper is as follows. Section 2 describes problem definition. Some related works are pointed in section 3 ; the proposed systems architecture is declared in section 4; and a sample run result is dedicated to section 5 . We finalize our work with a conclusion and future work part in section 6 .

\section{PROBLEM DEFINITION}

Let multi-graph $S=(P, R)$ represent a social network, where $P$ is the set of persons, and $R$ is the set of relationship indicating two persons participated in a certain event labeled by an ID $e_{i}$. Basically, this event with respect to the social network type can vary from email delivery, chatting history, or tagging a photo, to a police criminal record. Apparently such a multigraph contains number of cliques with different sizes representing participation in different events. Figure 1 shows dataset modeling for a small multi-graph.

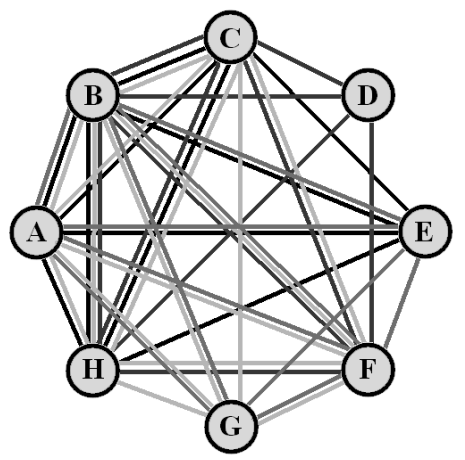

\begin{tabular}{|l|l|}
\hline$e_{0}$ & H C B E A \\
\hline$e_{1}$ & C B D H F \\
\hline$e_{2}$ & H C A B G F \\
\hline$e_{3}$ & G A F B E \\
\hline
\end{tabular}

Figure 1. One social network

DEFINITION 1. (Social network supergraph) Let $S^{\prime}=\left(P^{\prime}, R^{\prime}\right)$ be a supergraph in which $P^{\prime}$ is the set of supernodes and $R^{\prime}$ is the set of superedges. $\left|P^{\prime}\right|$ is equal to the number of events in the multi-graph. Each supernode $P^{\prime}{ }_{i}$ is assigned a label as the event ID, and its members are the nodes participated in the event. There exists a superedge between two supernodes if they have nodes in common and its weight represents the number of common nodes. Figure 2 shows the supergraph modeling of the social network multi-graph in Figure 1. 


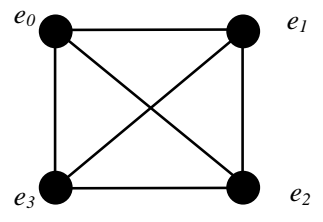

Figure 2. Transformed supergraph

DEFINITION 2. (Cooperation factor) In a supergraph $S^{\prime}=\left(P^{\prime}, R^{\prime}\right)$, the cooperation factor $(C F)$ between two supernodes $P^{\prime}{ }_{i}$ and $P^{\prime}{ }_{j}$ is defined as:

$$
C F\left(S^{\prime}, P_{i}^{\prime}, P_{j}^{\prime}\right)=\frac{2 \times \# \text { CommonNodes }\left(P_{i}^{\prime}, P_{j}^{\prime}\right)}{\# \text { TotalNodes }\left(P_{i}^{\prime}, P_{j}^{\prime}\right)} \quad ; C F \in[0,1]
$$

DEFINITION 3. (Cooperation distance metric) Having a supergraph $S^{\prime}=\left(P^{\prime}, R^{\prime}\right)$, and a list of $i$ person, denoted as a query $Q=\left\langle q_{1}, q_{2}, \ldots, q_{i}\right\rangle$, a query $Q=\langle q 1, q 2, \ldots, q i>$, we define the cooperation distance metric between query $Q$ and node $X^{*}$ as follows. Let $P_{i} \subset P^{\prime}$ be the set of supernodes in which $q i\left(i^{\text {th }}\right.$ member of $Q$ ) belongs to, and $U^{*}$ be the set of supernodes contain $X^{*}$. The cooperation distance $(C D)$ is then defined as:

$$
C D\left(S^{\prime}, P^{i}, P^{*}\right)=\frac{\sum_{i=1}^{|Q|} \text { ShortestPath }\left(S^{\prime}, P^{i}, P^{*}\right)}{|Q|}
$$

, where $\operatorname{ShortestPath}\left(S^{\prime}, P_{i}, P^{*}\right)$ is 0 if both $q i$ and $X^{*}$ belong to a same supernode; and otherwise is the output of a shortest graph path algorithm between nodes $\mathrm{Pi}$ and $\mathrm{P} *$ considering weight of superedges equal to $1-C F\left(S^{\prime}, P^{\prime}, P^{\prime *}\right)$. So for example if $X^{*}$ belongs to a supernode which all nodes in the $Q$ are in it, i.e., $\forall i=1 \ldots|Q| ;\left(P^{\prime}{ }_{i}, P^{\prime} *\right) \in R^{\prime}$ then $C D\left(S^{\prime}, X^{*}, Q\right)=0$.

\section{A. Problem}

Having a set of $n$ social networks $S_{i}=\left(P_{i}, R_{i}\right)$ and a list of $i$ person, could be arrested by police, denoted as a query $Q=<q_{1}$, $q_{2}, \ldots, q_{i}>$, we are interested in finding subsets of persons associated with the ones in $\mathrm{Q}$, and provide a fusion list, ordered by an increasing cooperation distance, which shows probability of having persons cooperated in a crime.

\section{B. Example}

For two social networks, S1 containing 8 events and 10 persons, $\mathrm{S} 1=\{\{\mathrm{P} 1, \mathrm{P} 6, \mathrm{P} 5, \mathrm{P} 3, \mathrm{P} 8, \mathrm{P} 0\},\{\mathrm{P} 2, \mathrm{P} 5, \mathrm{P} 9, \mathrm{P} 7\},\{\mathrm{P} 3$, P6, P8 $\},\{\mathrm{P} 4, \mathrm{P} 6, \mathrm{P} 3, \mathrm{P} 5\},\{\mathrm{P} 5, \mathrm{P} 4, \mathrm{P} 2, \mathrm{P} 3, \mathrm{P} 8\},\{\mathrm{P} 6, \mathrm{P} 4 \mathrm{P} 9$, $\mathrm{P} 1\},\{\mathrm{P} 7, \mathrm{P} 3, \mathrm{P} 5, \mathrm{P} 0, \mathrm{P} 8\},\{\mathrm{P} 8, \mathrm{P} 1\}\}$ and $\mathrm{S} 2$ containing 10 events and 8 persons, $\mathrm{S} 2=\{\{\mathrm{P} 1, \mathrm{P} 6, \mathrm{P} 8, \mathrm{P} 5\},\{\mathrm{P} 2, \mathrm{P} 4, \mathrm{P} 8\}$, $\{\mathrm{P} 3, \mathrm{P} 4, \mathrm{P} 7, \mathrm{P} 2\},\{\mathrm{P} 4, \mathrm{P} 8, \mathrm{P} 3\},\{\mathrm{P} 5, \mathrm{P} 1, \mathrm{P} 2\},\{\mathrm{P} 6, \mathrm{P} 8, \mathrm{P} 1\}$, $\{\mathrm{P} 7, \mathrm{P} 3, \mathrm{P} 1, \mathrm{P} 4, \mathrm{P} 8\},\{\mathrm{P} 8, \mathrm{P} 4, \mathrm{P} 3, \mathrm{P} 5, \mathrm{P} 7\},\{\mathrm{P} 1, \mathrm{P} 4, \mathrm{P} 7, \mathrm{P} 6, \mathrm{P} 3$, $\mathrm{P} 5, \mathrm{P} 8\},\{\mathrm{P} 2, \mathrm{P} 4, \mathrm{P} 1, \mathrm{P} 7\}\}$, and the query $\mathrm{Q}=\langle\mathrm{P} 1, \mathrm{P} 4, \mathrm{P} 7\rangle$, an ordered list of relevant results would be $\{\mathrm{P} 5, \mathrm{P} 3\},\{\mathrm{P} 5, \mathrm{P} 8$, $\mathrm{P} 3\},\{\mathrm{P} 5, \mathrm{P} 8\},\{\mathrm{P} 8, \mathrm{P} 3\},\{\mathrm{P} 5, \mathrm{P} 6, \mathrm{P} 8, \mathrm{P} 3\},\{\mathrm{P} 5, \mathrm{P} 6, \mathrm{P} 8\},\{\mathrm{P} 5$, $\mathrm{P} 6, \mathrm{P} 3\},\{\mathrm{P} 9, \mathrm{P} 6\}$, and etc., respectively as the cooperation distances for these subsets forms a semi-increasing sequence as 0.43122, 0.44214, 0.4509, 0.44425, 0.45477, 0.46486, 0.45170, 0.47378 .
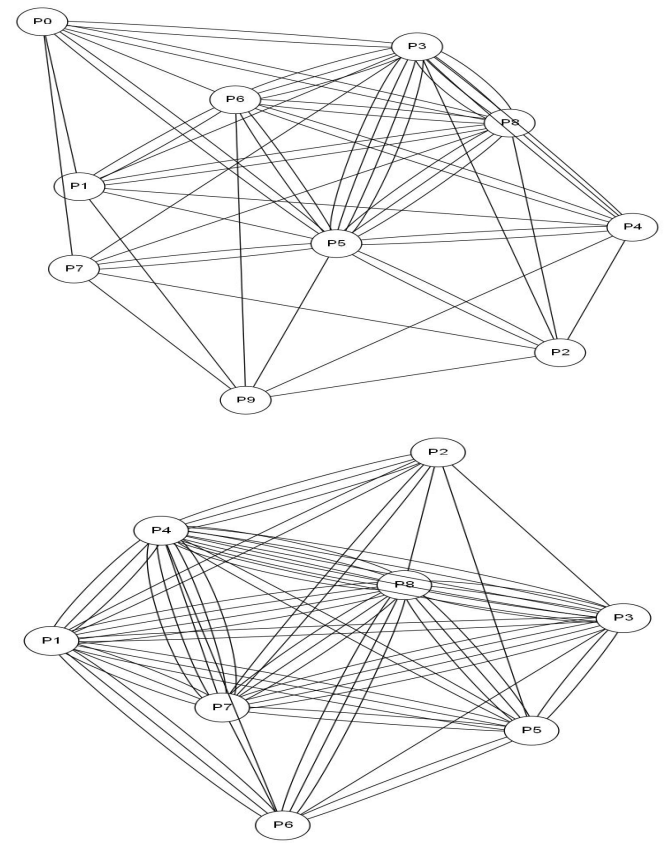

III. RELATED WORK

Many previous works in data mining have studied graph structured data analysis in different ways [6], [7], [8], [9]. Criminal network analysis as a specific case has also been a matter of concern in the past decade. In [10] a concept-space approach was used to extract criminal relations from the incident summaries and create a likely network of suspects. Co-occurrence weight measured the relational strength between two criminals by computing how frequently they were identified in the same incident. They used hierarchical clustering to partition the network into subgroups and the block-modeling approach to identify interaction patterns between these subgroups. They also calculated centrality measures of degree, betweenness, and closeness, to detect key members in each group. In another research [11] a framework for automated criminal network analysis and visualization was proposed that includes the major stages of a network analysis process: network creation, network partition, structural analysis, and network visualization. The authors also developed a system called CrimeNet Explorer, which has structural analysis functionality to detect subgroups, to identify between-group interaction patterns, and to identify central members of subgroups. However, their system has some limitations such as using a very simplistic concept space approach, only considering criminal-criminal relationships and not relations such as locations, weapons, vehicles, and organizations, and not considering a time dimension to the system. A tool was described in [12] which compiled a criminal profile out of the four important factors describing a criminal career for each individual offender: frequency, seriousness, duration and nature. These profiles were compared on similarity for all possible pairs of criminals using a new comparison method. They developed a specific distance measure to combine this profile difference with crime frequency and the change of criminal behavior over time to create a distance matrix that describes the amount of variation in criminal careers between all couples of perpetrators. 
In [13] authors provided mining cross-graph quasi-cliques technique which is generalized from several interesting applications such as cross-market customer segmentation and joint mining of gene expression data and protein interaction data. They solved a similar problem as ours in a sense of joint mining of multiple data sets to discover interesting, novel, and reliable patterns which cannot be obtained solely from any single source. The problem of mining cross-graph quasi-cliques is to find the complete set of cross-graph quasi-cliques that each has at least minimum size threshold vertices. For this \#Pcomplete ${ }^{1}$ problem, authors develop an efficient algorithm, Crochet, to mine cross-graph quasi-cliques. However, their work does not provide a ranking of subsets of related nodes with respect to a query of persons as in our case.

Social networks can be regarded as temporal interactions or event based cascading communication [14], [15]. Some works [16], [17], has theoretically studied communication times among nodes but not considering real-world datasets, while some extended such work by analyzing email datasets [18]. Recently we are enabled to get data about many aspects of human behavior such as data on people's use of the phone [19], people's mobility around a city [20] or a campus site [21], and interactions in a group [22]. Such available resources would help social network scholars to use emulations instead of simulations, for studies in the filed of social complex networks [23]. Related works are still in progress; however, previous ideas are not precisely proper for our defined problem. Lacking event-based idea, and not providing ranking for subsets results, motivated us for a new approach.

\section{PROPOSED METHOD}

We assume that there exists a relationship between two people if they have participated in the same event. Regarding the problem definition in section 2-A, at first glance it seems that the solution is an easy intersection finding among neighbors of the query node set in the graph, however, this can not provide us with a suitable suspicion list. In other words, such an algorithm just gives us a set of nodes without any order while here we are interested in having sorted list of subsets based on some probability. Here we are interested in returning sets of persons, not individual ones, and also we rank them in the final list. On the other hand providing all subsets of a big social network structure has a high complexity and seems inefficient to compute all CD metric for $2^{\mathrm{n}}$ subsets. Also in the context of social network analysis, Frequent Sub-graph Mining methods are not applicable for our problem model since we are not going to find any repeated clique pattern as each person is a unique entity in comparison to applications such as chemical networks or bioinformatics.

To solve the problem defined in section 2-A, we propose a three-phase algorithm, similar to our previous work [5]. The system described in [5] presents a hierarchical knowledge discovery process in a heterogeneous data grid environment.

${ }^{1} \mathrm{AP}$ is a complexity class which is the set of counting problems associated with the decision problems in the set NP. A problem is \#P-complete if and only if it is in \#P, and for any NP machine, the problem of computing its number of accepting paths can be reduced to this problem.[Wikipedia]
Although the proposed mechanism is suitable for multi-expert systems design, which was applied to build distributed diseases diagnosis system, some ideas such as game theory modeling of knowledge presentation and Dempster-Shafer combination approach come handy in our problem.

\section{A. Algorithm and system design}

At the first phase, by converting social network multi-graph into a relation of event-based transactions, as shown in figure 1 , we take advantage of association rules mining algorithm to provide frequent item sets. Note that frequent item sets mining process is different from frequent sub-graph mining as we mentioned earlier. In this step, frequent item sets help us producing rules for the next phase. In the second phase we take advantage of First Order Logic (FOL) structure for inference procedure since FOL is a suitable approach to model a knowledge-base and apply inference based on it. In a sense, this step provides us an inference engine to retrieve answer out of a query. To meet so we need to build a knowledge-base in which the query $Q$ (those who have been arrested by police in our special case) represent its Facts and extracted association rules represent its Rules. Then a forward chaining mechanism will give us some results from each social network. Finally in the third phase Dempster-Shafer theory of evidence would be applied to provide the fusion list ordered by relevancy.

Due to the distributed property of our problem (mining in multiple networks) and also need for parallel computing to fasten processing, we decided to use multi-agent architecture. Our proposed multi-agent system, depicted in figure 3, contains four different types of agents, each of which having its own characteristics as the followings:

Association Rules Miner Agent (ARMA): ARMAs are used to discover useful association rules and convert them to First Order Logic (FOL) to be included in the local IA knowledge base. The local IA is the one which is responsible for its local site ARM agents.

Broker Agent (BA): This agent will deliver the query from user to Inference Agents. The query is in the form of facts to be included in IA knowledge base.

Inference Agent (IA): Inference Agents use FOL based rules gathered by ARMAs, FOL based facts from BA, and apply the inference mechanism (here forward chaining) and return their inference result to the response agent.

Response Agent (RA): This agent is responsible for showing the result of retrieved information by collecting IAs results, combining them using Dempster-Shafer method, and then writing them on the screen ordered by relation percentage.

\section{B. ARM Agent Behavior}

ARM agents start rule mining by applying the well-known Apriori algorithm [24]. The algorithm computes the frequent itemsets in the database through several iterations. Iterations $i$ computes all frequent $i$-itemsets (itemsets with $i$ elements). Two main parameters here are MinSup and MinCon, which denote minimum acceptable support and confidence values respectively. 


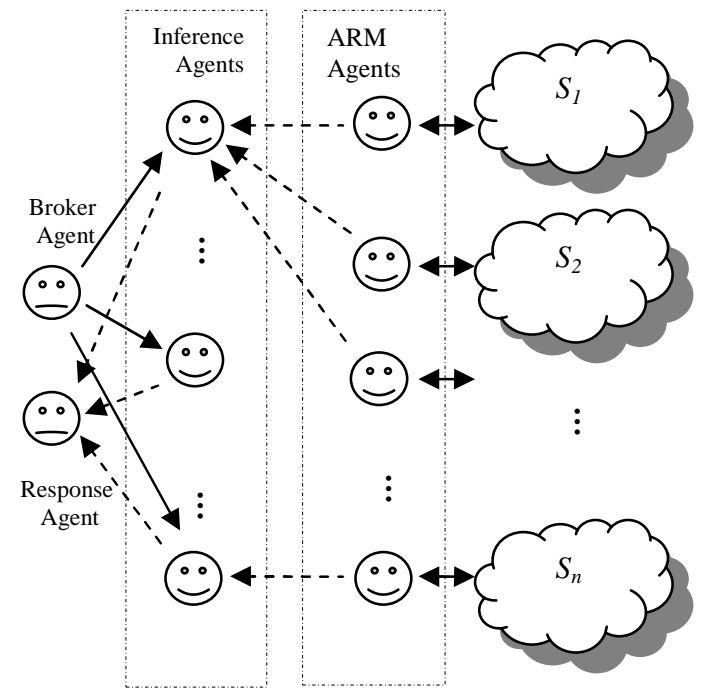

Figure 3. Multi-agent System Architecture

Since setting these parameters appropriately is known to be a hard problem, in most practical cases they are set to $50 \%$. In this work we show how to find a better threshold using a simple game theory mechanism. Game theory provides us with the mathematical tools to understand the possible strategies that utility-maximizing agents might use for decision making process. In an $n$-player games, the Nash equilibrium, is a set of strategies such that, given that for all I player $i$ plays strategy $s_{i}$, no player $j$ can get a higher payoff by playing a strategy other then $s_{j}$ [25]. A classic example for equilibrium point is two prisoners dilemma (PD), in which there are two prisoners kept in separated cells. If both confess they would be sentenced three years in jail. If one confesses and the other one does not, the first one goes four years in jail and another one would release, and if none of them confess they would stay in prison for a less important crime for one year. We will use the same idea to set MinCon and MinSup for ARMAs. Consider a game in which players aim at publishing at least one paper in a multiconference anyway possible. We also assume players are multi expert so they can publish in different fields. Also we know that in each field only a limited number of papers would be accepted. As a result each player tries to submit any paper in any field he can, and with any quality! So if another player does not present a paper in a particular field that the other one did, he would have $100 \%$ chance of acceptance in contrast with the one who did not proposed. However, if both players submit papers since we have no information about the quality, there would be an equal $50 \%$ chance for each player. Intuitively the game equilibrium would be the case in which each player submits any paper and with any quality. This scenario is somehow the same as our case. If each ARM agent only provides rules of associations among people in a social network with high MinSup, low support rules will be disregard while they might be very important in the final result since IA receives these rules and take the ones with the max support and confidence in case of same set of rules from different ARM agents. Thus in such a game model, ARM agent will not get any payoff if can not present any amount of knowledge about an association rule. This is considered by choosing a suitable MinCon and MinSup.
LEMMA 1. (Knowledge presentation equilibrium) [5] In a knowledge presentation game, there exists equilibrium in which players would select their knowledge with the highest possible confidence and support.

Proof. Let $A$ be the payoff for mutual knowledge presentation, $B$ the payoff for the presenter and $D$ for not-presenter in case one presents and the other does not, and $C$ the payoff when both do not present. In case $A$, both players receive a reward of $50 \%$ which is chance of presenting their work. In case $B$, the one who presented the knowledge would get $100 \%$ and the other player receives the payoff $0 \%$; and in case $C$ both players receive payoff $0 \%$. According to equilibrium condition in $\mathrm{PD}$, certain following conditions must hold: $B \geq A \geq C \geq D$ and the reward for $A$ should be greater than the average of the payoff for the $B$ and the $D$; i.e., the $A \geq(D+B) / 2$ and we see our model yields these equations; therefore it has equilibrium, as in PD. $\square$

This mechanism assures that all ARMAs propose extracted rules from transactions containing distinct itemset with any amount of sup and conf and if found one with higher sup and conf values, consider the new one. The ARMA game matrix in figure 4 shows agents payoff.

\begin{tabular}{|l|c|c|}
\hline & $\begin{array}{c}\text { Present } \\
\text { knowledge }\end{array}$ & $\begin{array}{c}\text { Do not present } \\
\text { knowledge }\end{array}$ \\
\hline Present knowledge & $50 \%-50 \%$ & $100 \%-0 \%$ \\
\hline Do not present knowledge & $0 \%-100 \%$ & $0 \%-0 \%$ \\
\hline
\end{tabular}

Figure 4. proposed ARMA game matrix

\section{Inference Agent Behavior}

The extracted association rules from ARMAs will be sent to IAs with their corresponding support and confidence. These rules plus the fact query sent by the BA will construct the IA knowledge base. By this time agents are able to inference results. There are two main methods of reasoning when using inference rules, forward chaining and backward chaining. Forward chaining is a data-driven method which starts with the available data and uses inference rules to extract more data until reaching a goal. Backward chaining, on the other hand, is goal-driven and starts with a list of goals and works backwards from the consequent to the antecedent to see if there is data available that support consequents. Expert systems often use backward chaining; however, due to our data-driven model, we used forward chaining. The forward chaining starts by adding new fact $F$ to the knowledge base and finds all conditional combinations having $F$ in assumption.

\section{Response Agent Behavior}

The response agent finally obtains all results from IAs and then combines them using Dempster-Shafer theory. These results will be then proposed to the user in an ordered list according to their relevancy. The Dempster-Shafer (DS) theory of evidence was first introduced by Dempster (1968) [26] and then extended by Shafer (1976) [27]. This theory is actually an extension to classic probabilistic uncertainty modeling. Whereas the Bayesian theory requires probabilities for each question of interest, belief functions allow us to base degrees of belief for one question on probabilities for a related question. In information retrieval the uncertainty occurs in three cases regarding relation of a document to a query: existence of 
different evidences, unknown number of evidences, or existence of incorrect evidences. In our case, we have different evidences form different social networks. In the DS theory, we assume a Universe of Discourse $\theta$, which is a set of mutually exclusive alternatives. In our case of determining the crime cooperator, $\theta$ would be the set of social network users. Now, Belief is a value for expressing certainty of a proposition and is calculated with respect to a density function $m: 2^{\theta} \rightarrow[0,1]$ called Basic Probability Assignment (BPA), where $m(A)$ represents Partial Belief amount of $\mathrm{A}$ and satisfies the followings: $m(\varnothing)=0$ and $\sum_{\mathrm{A} \in \mathrm{U}} m(A)=1 . A$ is called a focal element, which with the associated BPA defines a body of evidence. To measure the Total Belief amount of $A \subset U$ the belief function is defined as: $\operatorname{Bel}(A)=\sum_{\forall \mathrm{B} \subset \mathrm{A}} m(B)$. Shafer defined doubt amount in $A$ as the belief in $A^{\prime}$, and the Plausibility function as the total belief amount in $A$, where $\operatorname{Pl}(A)$ is actually the high boundary of belief in $A$ so that the correct belief in $A$ is in the interval of $[\operatorname{Bel}(A), P l(A)]$. Doubt function is defined as: $\operatorname{Dou}(A)=\operatorname{Bel}\left(A^{\prime}\right)$.

$$
P l(A)=1-\operatorname{Dou}(A)=\sum_{B \subseteq U} m(B)-\sum_{B \subseteq A^{\prime}} m(B)=\sum_{B \cap A \neq \phi} m(B)
$$

Dempster's rule of combination is a generalization of Bayes' rule which emphasizes the agreement between multiple sources and ignores all the conflicting evidence by a normalization factor. Since confidence value in association rules implies how reliable a rule is, we can consider the normalized value of the confidence as a partial belief amount and therefore the BPA for the focal elements, which are the users set, in our model are:

$$
m\left(A_{i}\right)=\operatorname{Conf}\left(A_{i}\right) / \sum_{i \in 2^{\theta}} \operatorname{Conf}\left(A_{i}\right)
$$

Let $m_{l}$ and $m_{2}$ are the BPAs in a frame of discernment. The combination $\mathrm{BPA}$ is then calculated in the following manner:

$$
m(A)=m_{1} \otimes m_{2}=\frac{\sum_{B \cap C=A} m_{1}(B) m_{2}(C)}{\sum_{B \cap C \neq \varnothing} m_{1}(B) m_{2}(C)}
$$

\section{EXPERIMENTAL RESULTS}

Since we did not have access to real-world criminal interaction dataset, instead we take advantage of similar collaborations but among professors and students. In order to show the mining process in our system, we used SBNS Datasets from the Auton Laboratory of the School of Computer Science in Carnegie Mellon University ${ }^{2}$. We chose Institute Data, consist of set of records of collaborations between professors and students collected from publicly available web pages listed on Carnegie Mellon University Robotics Institute's web site. The dataset contains 3,342 people, 5,152 recors, 2.77 Avg people/record, and 4.24 Avg record/person. To simulate multiple social networks, we divided these records into two datasets containing $1 / 3$ and $2 / 3$ of original records. After extracting association rules by ARMAs, knowledge bases would be generated by the IAs. In the next step, the broker agent delivers queries Q1, Q2, and Q3 separately to each IAs as facts to be added in the knowledge base:

$$
Q 1=<\text { Zachary_Omohundro, Aaron_Christopher_Morris > }
$$

\footnotetext{
${ }^{2}$ http://www.autonlab.org/autonweb/downloads/datasets.html
}

\section{$Q 2=<$ Christopher_Baker, Sebastian_Thrun, Dirk_Haehnel $>$ \\ $Q 3=<$ Nancy_Pollard, Jessica_K_Hodgins $>$}

Our two IAs use forward chaining and send the two result sets to the RA. The 5 results out of 80 selected rules by IA\#1 and the 5 ones out of 32 selected by the IA\#2 for the Q1, are depicted in figure 5. ( $s$ : association support, $c$ : association confidence, $m$ : partial belief amount of the association)

\begin{tabular}{l}
\hline IA\#1 \\
\hline Aaron_Christopher_Morris(s:0.00114,c:0.8),m=0.014285714 \\
Christopher_Baker(s:0.00114,c:0.8),,m=0.014285714 \\
Christopher_Baker,Chuck_Whittaker(s:0.00114,c:0.4),m=0.007142857 \\
Christopher_Baker,Chuck_Whittaker,William_Red_L._Whittaker(s:0.00114,c \\
$: 0.4), m=0.007142857$ \\
Christopher_Baker,Sebastian_Thrun(s:0.00114,c:0.8),m=0.014285714 \\
... IA\#2 \\
\hline Christopher_Baker(s:0.00114,c:1.0),m=0.038461538 \\
Christopher_Baker,Chuck_Whittaker(s:0.00114,c:0.8),m=0.030769231 \\
Christopher_Baker,Chuck_Whittaker,William_Red_L._Whittaker(s:0.00114,c \\
$: 0.8), m=0.030769231$ \\
Christopher_Baker,William_Red_L._Whittaker(s:0.00114,c:0.8),m=0.030769 \\
231 \\
Chuck_Whittaker(s:0.00142,c:0.5),m=0.019230769 \\
$\ldots$
\end{tabular}

Figure 5. Inference Agents Output

Finally RA implies D-S combination and returns the final result. The final top 15 results in ranking list with respect to BPA values for Q1 is shown in Table 1. The graph in figure 6, shows average ranking for these three queries with respect to cooperation distance metric which indicates our system provides reasonable ranking based on the proposed approach and our defined metric.

\section{CONCLUSION AND FUTURE WORK}

A new social network mining method for analyzing criminal groups was investigated in term of using social relation data. The idea varies in different ways from previous works such as mining from multiple sources, generic event modeling, and providing a ranked result fusion list. We proposed a multi-agent architecture involving game-theory modeling for competitive knowledge extraction and DempsterShafer inference combination. A simulation was done on a real-world dataset and the final ranked list with respect to cooperation distance indicates our system provides a reasonable ranking based on the proposed approach and the defined metric. In this work, we just focus on multi-graphs with events as label however a future work can be a real temporal graph considering sequence of incidents.

\section{ACKNOWLEDGMENT}

Authors would like to thank Mohsen Jamali for his valuable comments on improving parts of this work, and five anonymous reviewers for their helpful feedback. 
TABLE I. TOP 15 RESULTS FOR Q1

\begin{tabular}{|c|c|c|}
\hline Results & $\begin{array}{l}\text { BPA from } \\
\text { D-S }\end{array}$ & CD metric \\
\hline Christopher_Baker,William_Red_L._Whittaker & 0.0159691 & 9.757035 \\
\hline David_Ferguson,William_Red_L._Whittaker & 0.014653 & 9.79319 \\
\hline $\begin{array}{c}\text { Scott_Thayer,Chuck_Whittaker,William_Red_L } \\
\text {._Whittaker }\end{array}$ & 0.0137756 & 9.75994 \\
\hline $\begin{array}{c}\text { Scott_Thayer,Christopher_Baker,Chuck_Whitta } \\
\text { ker,William_Red_L._Whittaker }\end{array}$ & 0.0137756 & 9.76382 \\
\hline $\begin{array}{c}\text { Scott_Thayer,David_Ferguson,Chuck_Whittaker } \\
\text {,William_Red_L._Whittaker }\end{array}$ & 0.0137756 & 9.7968975 \\
\hline $\begin{array}{c}\text { Scott_Thayer,Christopher_Baker,William_Red_ } \\
\text { L._Whittaker }\end{array}$ & 0.0137756 & $\begin{array}{l}9.7979866 \\
67\end{array}$ \\
\hline $\begin{array}{c}\text { Christopher_Baker,Chuck_Whittaker,William_R } \\
\text { ed_L._Whittaker }\end{array}$ & 0.0137756 & 9.80513 \\
\hline $\begin{array}{c}\text { Scott_Thayer,David_Ferguson,Christopher_Bak } \\
\text { er,William_Red_L._Whittaker }\end{array}$ & 0.0137756 & 9.8254325 \\
\hline $\begin{array}{l}\text { David_Ferguson,Christopher_Baker,Chuck_Whi } \\
\text { ttaker,William_Red_L._Whittaker }\end{array}$ & 0.0137756 & 9.83079 \\
\hline $\begin{array}{c}\text { Scott_Thayer,David_Ferguson,William_Red_L. } \\
\text { _Whittaker }\end{array}$ & 0.0137756 & 9.84209 \\
\hline $\begin{array}{c}\text { David_Ferguson,Chuck_Whittaker,William_Red } \\
\text { _L._Whittaker }\end{array}$ & 0.0137756 & $\begin{array}{c}9.8492333 \\
33\end{array}$ \\
\hline $\begin{array}{c}\text { David_Ferguson,Christopher_Baker,William_Re } \\
\text { d_L._Whittaker }\end{array}$ & 0.0137756 & 9.88728 \\
\hline Scott_Thayer,William_Red_L._Whittaker & 0.0120207 & 9.80925 \\
\hline Chuck_Whittaker,William_Red_L._Whittaker & 0.0120207 & 9.819965 \\
\hline William_Red_L._Whittaker & 0.0120207 & 9.97861 \\
\hline
\end{tabular}

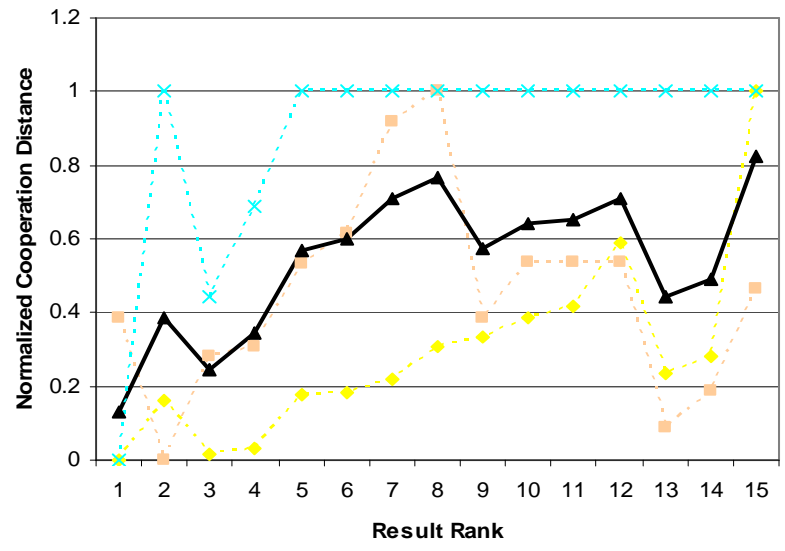

Figure 6. Ranking with respect to cooperation distance

\section{REFERENCES}

[1] S. H. Strogatz, "Exploring complex networks", Nature 410, 2001, pp. 268-276.

[2] P. Holme, "Network dynamics of ongoing social relationships", Europhysics Letters 64 (3), 2003, pp. 427-433.

[3] P.Holme, S.M. Park, B.J. Kim, and C.R. Edling, "Korean university life in a network perspective", Dynamics of a large affiliation network, Physica A 307, 2007, pp. 821-830.

[4] A.-L., Barabasi, R. Albert, "Emergence of scaling in random networks", Science 286, 1999, pp. 509-512.
[5] A. Milani Fard, "Competitive-Cooperative Automated Reasoning from Distributed and Multiple Source of Data", in: Data Mining and Multiagent Integration, Ed: Longbing Cao, LNCS 5680, Springer, 2009

[6] K. Borgwardt, X. Yan: "Graph Mining and Graph Kernels”, KDD, 2008

[7] J. Kleinberg, et al.: "The web as a graph: Measurements, models and methods", COCOON, 1998

[8] J. Leskovec and C. Faloutsos, "Mining Large Graphs", Tutorial ECML/PKDD, 2007

[9] M. E. J. Newman: "Fast algorithm for detecting community structure in networks", Phys. Rev. E 69, 2004

[10] H. Chen, W. Chung, J. J. Xu, G. Wang, Y. Qin, M. Chau, "Crime Data Mining: A General Framework and Some Examples", Computer, vol. 37, no. 4, 2004, pp. 50-56

[11] J. J. Xu, H. Chen, "CrimeNet explorer: a framework for criminal network knowledge discovery" ACM Trans. Inf. Syst., Vol. 23, No. 2., 2005, pp. 201-226.

[12] J. S. de Bruin, T. K. Cocx, W. A. Kosters, J. F. J. Laros, and J. N. Kok, "Data Mining Approaches to Criminal Career Analysis", Sixth IEEE International Conference on Data Mining ICDM'06, 2006, pp. 171-177

[13] J. Pei, D. Jiang, and A. Zhang. "On Mining Cross-Graph QuasiCliques". In Proceedings of the 11th ACM SIGKDD (KDD'05), Chicago, IL, USA, August 21-24, 2005

[14] E. Adar, L. Zhang, L.A. Adamic, R.M. Lukose, Implicit structure and the dynamics of blogspace, in: Workshop on the Weblogging Ecosystem, 2004.

[15] J. Leskovec, M. McGlohon, C. Faloutsos, N. Glance, M. Hurst, Cascading behavior in large blog graphs, in: Proc. SIAM International Conference on Data Mining, 2007.

[16] E. Cheng, J.W. Grossman and M.J. Lipman, Time-stamped graphs and their associated influence digraphs, Discrete Applied Mathematics 128 2003, pp. 317-335.

[17] D. Kempe, J. Kleinberg, A. Kumar, Connectivity and inference problems for temporal networks, in Proc. 32nd ACM Symp. on Theory of Computing, 2000, pp. 504-513.

[18] P. Holme, Network reachability of real-world contact sequences, Physical Review E 71, 2005, p. 046119.

[19] J.-P. Onnela, J. Saramäki, J. Hyvönen, G. Szabó, D. Lazer, K. Kaski, J. Kertesz and A.-L. Barabási, Structure and tie strengths in mobile communication networks, Proceedings of the National Academy of Sciences 104, 2007, pp. 7332-7336. Full Text via CrossRef | View Record in Scopus | Cited By in Scopus (26)

[20] M.C. Gonzalez, C.A. Hidalgo, A.-L. Barabási, Understanding individual humanmobility patterns, Nature 453, 2008, pp. 479-482.

[21] M. Balazinska and P. Castro, Characterizing mobility and network usage in a corporate wireless local-area network, MobiSys '03 Proceedings First International Conference on Mobile Systems, Applications and Services, ACM Press, New York, 2003, pp. 303-316.

[22] A. Chaintreau, P. Hui, J. Crowcroft, C. Diot, R. Gass and J. Scott, Impact of human mobility on the design of opportunistic forwarding algorithms, Proceedings 25th IEEE Conference on Computer Communications (INFOCOM), IEEE CS Press, New York, 2006.

[23] E. Yoneki, P. Hui and J. Crowcroft, Wireless epidemic spread in dynamic human networks, Bio-Inspired Computing and Communication, LNCS vol. 5151, Springer, 2008, pp. 116-132.

[24] Agrawal R, T. Imielinski, A.N. Swami, "Mining Association Rules between Sets of Items in Large Databases.” SIGMOD, 22(2): June 1993.

[25] A.; Neyman, "Bounded complexity justifies cooperation in finitely repeated prisoner's dilemma". Economic Letters, 1985, pages 227-229.

[26] Dempster, A., A generalization ofba yesian inference. Journal of the Royal Statistical Society, 1968, 30:205-247.

[27] Schafer, G., A mathematical theory of evidence, volume 2702. Princetown University Press, 1976. 